\title{
Distributed Consensus Design for a Class of Uncertain Linear Multiagent Systems under Unbalanced Randomly Switching Directed Topologies
}

\author{
Dinh Hoa Nguyen (iD) \\ WPI International Institute for Carbon-Neutral Energy Research, and Institute of Mathematics for Industry, \\ Kyushu University, Fukuoka, Japan \\ Correspondence should be addressed to Dinh Hoa Nguyen; hoa.nd@i2cner.kyushu-u.ac.jp
}

Received 23 May 2018; Revised 18 July 2018; Accepted 30 July 2018; Published 9 August 2018

Academic Editor: Carlos-Renato Vázquez

Copyright (C) 2018 Dinh Hoa Nguyen. This is an open access article distributed under the Creative Commons Attribution License, which permits unrestricted use, distribution, and reproduction in any medium, provided the original work is properly cited.

\begin{abstract}
This paper proposes a novel approach to design fully distributed consensus controllers for heterogeneous linear Multiagent Systems subjected to randomly switching directed topologies and model uncertainties. The appealing features of this approach are as follows. First, it uses the mildest assumption for the randomly switching topologies that the union of switched graphs has a spanning tree. Second, the consensus is achieved under a class of state multiplicative uncertainties. Moreover, the proposed consensus controllers are low-rank and have nonconservative coupling strengths. Finally, a numerical example is presented to illustrate the effectiveness of the proposed theoretical approach.
\end{abstract}

\section{Introduction}

Multiagent systems (MASs) have been widely studying from both theoretical viewpoints and practical applications, e.g., power networks, transportation systems, and autonomous vehicle groups, where a set of dynamical systems collaborate with their few neighbors to achieve their own targets as well as the whole system's objective. One of the most important and extensively investigated area in MASs is the consensus problem where agents' states or outputs reach a nonzero agreement [1-5].

Most of the studies so far on consensus of linear MASs with generic models of agents require solving Linear Matrix Inequality (LMI) problems and have a lower bound on the coupling strength depending on the eigenvalue with smallest nonzero real part of the graph Laplacian matrix, e.g., [6-8]. Therefore, the associated consensus algorithms are not fully distributed, and other algorithms to approximate eigenvalues of Laplacian matrix or to adjust the coupling strength in an adaptive way are needed. This unexpectedly increases the complexity and the computational efforts of consensus algorithms and possibly slows down the convergence to consensus. Another direction is to treat the agents from the passivity framework, e.g., [9], but additional conditions on the system matrices are needed, which limits the class of agents. Other works may utilize higher derivatives of agents' states, e.g., [10], which are not possible in many practical situations.

The studies for consensus of MASs under switching topologies often employ strong assumptions on each switched graph, such as strongly connectedness (e.g., [7]), or balance (e.g., [8, 11]), or both (e.g., [2, 9]). Those assumptions for deterministically switching graphs can be relaxed to be weaker, e.g., the switched undirected graphs are jointly connected [1]; the union of switched directed graphs remains strongly connected after sometime [12] or has a spanning tree frequently enough $[10,13]$. On the other hand, the relaxed assumptions for randomly switching graphs still require the balance of the union of switched graphs, e.g., [14]. In fact, consensus can be achieved under randomly switching topologies without that assumption. Thus, further investigations are emergent to overcome this limitation.

Linear Quadratic Regulator (LQR), a classical control design method, has been proven to be an effective approach for developing consensus algorithms [15-21], which usually 
results in solving structured Riccati equations. As previously shown, e.g., in $[19,21]$ and references therein, low-rank consensus design can reduce the computational cost and possibly increases the consensus speed. Furthermore, by employing the idea of selective pole shift to LQR, we have recently proposed a fully distributed approach in [21] to achieve not only a low-rank consensus controller but also a nonconservative consensus coupling strength for general linear MASs. Unfortunately, the communication graph in [21] is limited to be undirected and fixed. These results were then extended in [14] to both fixed and randomly switching directed graphs and also to handle state matrix uncertainty. However, union of switching graphs in [14] was assumed to be balanced and a single zero eigenvalue of system matrix of agents' dynamics was also assumed. This research will relax those assumptions to be less restrictive.

Most importantly, the current study proposes a fully distributed consensus design for heterogeneous linear MASs with multiplicative state uncertainty and randomly switching topologies where none of them is balanced or has a spanning tree and their union is unbalanced. This problem has not been addressed in the literature, to the best of our knowledge, and hence clearly constitutes the main contribution of the current research. Another contribution is that the proposed consensus controller has low rank and nonconservative coupling strength.

The following notations and symbols will be used in the paper. The notations $\mathbb{R}, \mathbb{C}, \mathbb{C}_{-}^{0}$, and $\mathbb{C}_{-}$stand for the sets of real, complex, complex with nonpositive real part, and complex with negative real part numbers. Moreover, $\mathbf{1}_{n}$ and $\mathbf{0}_{n}$ denote the $n \times 1$ vector with all elements equal to 1 and 0 , respectively, and $I_{n}$ denotes the $n \times n$ identity matrix. Next, $\operatorname{Re}(x)$ denotes the real part of a complex number $x$ and $\otimes$ stands for the Kronecker product. On the other hand, $\lambda(A)$ and $\lambda_{\text {min }}(A)$ denote the eigenvalue set and the eigenvalue with smallest, nonzero real part of $A$, respectively. Lastly, $>$ and $\succeq$ denote the positive definiteness and positive semidefiniteness of a matrix.

\section{Problem Description}

2.1. Graph Theory. Denote $(\mathscr{G}, \mathscr{V}, \mathscr{E})$ the directed graph representing the communication structure in an MAS composing of $N$ agents, where $\mathscr{V}$ and $\mathscr{E}$ represent the set of vertices and edges of $\mathscr{G}$, respectively. There is an edge $e_{i j} \in \mathscr{E}$ if agent $i$ receives information from agent $j$. The neighboring set of a vertex $i$ is denoted by $\mathcal{N}_{i} \triangleq\left\{j: e_{i j} \in \mathscr{E}\right\}$. Moreover, let $a_{i j}$ be elements of the adjacency matrix $\mathscr{A}$ of $\mathscr{G}$; i.e., $a_{i j}>0$ if $e_{i j} \in \mathscr{E}$ and $a_{i j}=0$ if $e_{i j} \notin \mathscr{E}$. The in-degree of a vertex $i$ is denoted by $\operatorname{deg}_{i}^{\text {in }} \triangleq \sum_{j=1}^{N} a_{i j}$. Let $\mathscr{D} \triangleq \operatorname{diag}\left\{\operatorname{deg}_{i}^{\text {in }}\right\}_{i=1, \ldots, N}$. Then $\mathscr{L} \triangleq \mathscr{D}-\mathscr{A}$ defines the Laplacian matrix. The out-degree of a vertex $i$ is denoted by $\operatorname{deg}_{i}^{\text {out }} \triangleq \sum_{j=1}^{N} a_{j i}$. Then $\mathscr{G}$ is said to be balanced if $\operatorname{deg}_{i}^{\text {in }}=\operatorname{deg}_{i}^{\text {out }} \forall i=1, \ldots, N$. A directed path connecting vertices $i$ and $j$ in $\mathscr{G}$ is a set of consecutive edges starting from $i$ and stopping at $j$. Then $\mathscr{G}$ is said to have a spanning tree if there exists a node from which there are directed paths to every other node.
Lemma 1 (see [13]). The Laplacian matrix $\mathscr{L}$ always has a zero eigenvalue with associated eigenvector $\mathbf{1}_{N}$, and all nonzero eigenvalues of $\mathscr{L}$ have positive real parts. Furthermore, $\mathscr{L}$ has only one zero eigenvalue if and only if $\mathscr{G}$ has a spanning tree.

The communication among agents considered in this research is randomly switched, which can be described by a continuous-time Markov process defined as follows. A continuous-time Markov process $\left\{\sigma_{t}: t \geq 0\right\}$, which takes values on the set $\mathbb{N}=\{1,2, \ldots$,$\} , is defined on the probability$ space $(\Omega, \mathscr{F}, \mathbb{P})$ where $\Omega$ is the space of elementary events, $\mathscr{F}$ is the underlying $\sigma$-field on $\Omega$, and $\mathbb{P}$ is a probability measure on $\mathscr{F}$. Let the infinitesimal generator be defined as follows $[8,22]$ :

$$
\begin{aligned}
& P(\sigma(t+h)=j \mid \sigma(t)=i) \\
& \quad= \begin{cases}q_{i j} h+o(h), & i \neq j, \\
1+q_{i i} h+o(h), & i=j,\end{cases}
\end{aligned}
$$

where $o(\cdot)$ stands for the Little-o notation and $q_{i j}$ represents the transition rate from state $i$ to state $j$ with $q_{i j} \geq 0$ as $i \neq$ $j$ and $q_{i i}=-\sum_{j \neq i} q_{i j}$. Then the transition rate matrix $\mathbb{Q}$ is defined by $\mathbb{Q} \triangleq\left[q_{i j}\right]$. It can be easily seen that the row sums of $\mathbb{Q}$ are zero.

Next, denote $\mathscr{G}(t)$ the randomly switching graph which switches among the elements of a finite set $\mathcal{S}_{\mathscr{G}} \triangleq$ $\left\{\mathscr{G}_{1}, \ldots, \mathscr{G}_{\ell}\right\}$, with a switching signal $\sigma(t) \in\{1, \ldots, \ell\}$, and $\mathscr{L}(t)$ the Laplacian matrix associated with $\mathscr{G}(t)$. Also, the time index $t$ is used for all parameters related to the timevarying graph $\mathscr{G}(t)$ such as $a_{i j}(t)$ and $\mathcal{N}_{i}(t)$. The transition rate matrix $\mathscr{Q}=\left[q_{i j}\right] \in \mathbb{R}^{\ell \times \ell}$. For $k=1, \ldots, \ell$, let $\mathscr{L}_{k}$ be the Laplacian matrix corresponding to the graph $\mathscr{G}_{k}$. Denote $\pi=\left[\pi_{1}, \ldots, \pi_{\ell}\right]^{T}$ the stationary distribution of this Markov process which is assumed to be ergodic. Thus, $\pi$ is unique, $\pi_{k}>0 \forall k=1, \ldots, \ell$, and each state of the Markov chain can be reached from any other state. Furthermore, we can also assume that the Markov process starts from $\pi$ [8]. As a result, the distribution of $\sigma(t)$ is equal to $\pi$ for all $t \geq 0$. Let $\pi^{*}=\min _{k=1, \ldots, \ell} \pi_{k}$. Finally, let $\mathscr{G}_{U} \triangleq \bigcup_{k=1, \ldots, \ell} \mathscr{G}_{k}$ denote the union of all possible switching topologies and $\mathscr{L}_{U}$ denote its Laplacian matrix.

2.2. Multiagent System Dynamics. Consider a class of MASs composing of $N$ linear agents subjected to multiplicative parametric uncertainties,

$$
\dot{x}_{i}=A\left(I_{n}+\Delta A_{i}\right) x_{i}+B u_{i}, \quad i=1, \ldots, N,
$$

where $x_{i} \in \mathbb{R}^{n}, u_{i} \in \mathbb{R}^{m}$ are vectors of states and control inputs of agent $i ; A \in \mathbb{R}^{n \times n}, B \in \mathbb{R}^{n \times m}$ are nominal state matrices; $\Delta A_{i} \in \mathbb{R}^{n \times n}$ represent the state uncertainties. Note that due to the uncertainties $\Delta A_{i}$ agents' dynamics is heterogeneous.

In literature, a widely used consensus protocol for the linear MASs in (2) without uncertainties (see, e.g., [2-4, 20, 21]) is

$$
u_{i}=-\mu K \sum_{j \in \mathscr{N}_{i}(t)} a_{i j}(t)\left(x_{i}-x_{j}\right), \quad i=1, \ldots, N,
$$


where $K \in \mathbb{R}^{m \times n}$ is a consensus controller gain matrix and $\mu>0$ is called the coupling strength. In this research, we also employ (3) for the uncertain MAS (2) and aim at finding $K$ and $\mu$ such that the consensus of agents is achieved, which is defined below.

Definition 2. The linear MAS (2) with a randomly switching topology $\mathscr{G}(t)$ is said to reach a mean-square consensus for any initial condition of agents and any initial distribution of the continuous-time Markov process if there exists $x^{*} \in \mathbb{R}^{n}$ such that

$$
\lim _{t \rightarrow \infty} \mathbb{E}\left[\left\|x_{i}(t)-x^{*}\right\|^{2}\right]=0, \quad \forall i=1, \ldots, N .
$$

Next, the following assumptions are employed, where assumptions (A2) and (A4) are for a finite and nonzero consensus state and (A2) is inspired by [23].

(A1): $(A, B)$ is stabilizable.

(A2): $A$ has a zero eigenvalue whose algebraic and geometric multiplicity are the same and equal to $\alpha$, while other eigenvalues of $A$ are in $\mathbb{C}_{-}$.

(A3): $\mathscr{G}_{\cup}$ has a spanning tree.

(A4): $\lambda\left(A\left(I_{n}+\Delta A_{i}\right)\right) \subset \mathbb{C}_{-}^{0}$.

Remark 3. Assumption (A3) is the mildest one for consensus with randomly switching directed graphs, which significantly and obviously advances the existing results in the literature that require stronger assumptions such as the balance of every switched graph $\mathscr{G}_{k} \forall k=1, \ldots, \ell$ (see, e.g., $[6,8]$ ), or the balance of $\mathscr{G}_{\cup}[14,24]$. On the other hand, assumption (A4) is made to be consistent with assumption (A2) and the latter consensus design and proof. This is an assumption to simplify the consensus design, and if it is not satisfied then another consensus design must be sought. Further, a bound of the uncertainty $\Delta A_{i}$ is usually known; hence tools from robust control theory can be utilized to express the assumption (A4) in the form of an LMI.

\section{Consensus Design for Unbalanced Randomly Switching Graphs and Model Uncertainty}

3.1. Leaderless Consensus Design. Let $P \in \mathbb{R}^{n \times n} P \succeq 0$ be the unique solution of the following Riccati equation:

$$
P A+A^{T} P+Q-P B R B^{T} P=0
$$

where $R \in \mathbb{R}^{m \times m}, R>0$ and $Q \in \mathbb{R}^{n \times n}$, and $Q \geq 0$ are design parameters and $\left(Q^{1 / 2}, A\right)$ is detectable. Let $\vee \in \mathbb{R}^{\alpha \times n}$ be the matrix whose rows are left eigenvectors associated with the zero eigenvalue of $A$. Due to assumption $(\mathrm{A} 2), \operatorname{rank}(\mathrm{V})=$ $\alpha$. Consequently, it can be shown that $P$ must have the form $P=\mathrm{V}^{T} \widehat{P} \bigvee$, where $\widehat{P} \in \mathbb{R}^{\alpha \times \alpha}, \widehat{P} \geq 0$, with $Q$ selected to be $\mathrm{Q}=\mathrm{V}^{T} \widehat{\mathrm{Q}} \mathrm{V}, \widehat{\mathrm{Q}} \in \mathbb{R}^{\alpha \times \alpha}, \widehat{Q} \geq 0$. This can be proved using the similar method in [21], so we skip here for brevity. Then the main result of this research is presented in the following theorem.
Theorem 4. Let $R=I_{m}$ and $\beta>0$ such that $\widehat{Q} \geq \beta \widehat{P}$. Under assumptions (A1)-(A4), the uncertain MAS (2) reaches a mean-square consensus by the distributed rank- $\alpha$ controller, for any $\mu>0, i=1, \ldots, N$,

$$
u_{i}(t)=-\mu B^{T} \bigvee^{T} \widehat{P} \bigvee \sum_{j \in \mathcal{N}_{i}(t)} a_{i j}(t)\left(x_{i}(t)-x_{j}(t)\right)
$$

Furthermore, the consensus speed is lower bounded by

$$
\pi^{*} \mu \beta \lambda_{\min }\left(\mathscr{L}_{U}+\mathscr{L}_{U}^{T}\right)
$$

Proof. The basic idea here is similar to that in [8], but significant difficulties need to be resolved upon system uncertainties and unbalanced switched graphs. Moreover, the consensus state is also different from that in [8] due to the unbalanced union graph.

Let $\zeta \in \mathbb{R}^{N}$ be such that $\zeta^{T} \mathscr{L}_{U}=0$ and $\mathbf{1}_{N} \zeta^{T}=1$. Denote $x^{*}(t) \triangleq \zeta^{T} x(t) ; \delta_{i}(t) \triangleq x_{i}(t)-x^{*}(t), i=1, \ldots, N ; \delta(t) \triangleq$ $\left[\delta_{1}(t)^{T}, \ldots, \delta_{N}(t)^{T}\right]^{T}$. Then $\delta(t)=\left[\mathscr{M} \otimes I_{n}\right] x(t)$, where $\mathscr{M} \triangleq$ $I_{N}-\mathbf{1}_{N} \zeta^{T}$.

Denote $\Delta A \triangleq \operatorname{diag}\left\{\Delta A_{i}\right\}_{i=1, \ldots, N}$. Since $\mathscr{L} \mathscr{M}=\mathscr{L}$, substituting (6) into (2) gives us

$$
\begin{aligned}
\dot{\delta}(t)= & {\left[I_{N} \otimes A-\mu(\mathscr{M} \mathscr{L}) \otimes\left(B B^{T} \mathrm{~V}^{T} \widehat{P} \mathrm{~V}\right)\right] \delta(t) } \\
& +[(\mathscr{M} \otimes A) \Delta A] x(t) \\
= & {\left[I_{N} \otimes A-\mu(\mathscr{M} \mathscr{L}) \otimes\left(B B^{T} \mathrm{~V}^{T} \widehat{P} \mathrm{~V}\right)\right] \delta(t) } \\
& +(\mathscr{M} \otimes A) \Delta A x(t) .
\end{aligned}
$$

Hence, $\delta(t)^{T}\left(I_{N} \otimes P\right) \dot{\delta}(t)=-\mu \delta(t)^{T}[(\mathscr{M} \mathscr{L}) \otimes$ $\left.\left(P B B^{T} \bigvee^{T} \widehat{P} \bigvee\right)\right] \delta(t)$, since $P A=\bigvee^{T} \widehat{P} \bigvee A=0$. Next, consider the following nonnegative quadratic functions,

$$
\begin{aligned}
V(t) & =\mathbb{E}\left[\delta(t)^{T}\left(I_{N} \otimes P\right) \delta(t)\right], \\
V_{k}(t) & =\mathbb{E}\left[\delta(t)^{T}\left(I_{N} \otimes P\right) \delta(t) 1_{\{\sigma(t)=k\}}\right],
\end{aligned}
$$

$$
k=1, \ldots, \ell,
$$

where $1_{\{\bullet\}}$ is the Dirac measure. Obviously, $V(t)=\sum_{k=1}^{\ell} V_{k}(t)$. Then using Lemma 4.2 in [22], we have

$$
\begin{aligned}
& d V_{k}(t)=\mathbb{E}\left[(d \delta(t))^{T}\left(I_{N} \otimes P\right) \delta(t) 1_{\{\sigma(t)=k\}}\right. \\
& \left.\quad+\delta(t)^{T}\left(I_{N} \otimes P\right) d \delta(t) 1_{\{\sigma(t)=k\}}\right]+\sum_{j=1}^{\ell} q_{j k} V_{j}(t) d t \\
& \quad+o(d t) .
\end{aligned}
$$


Therefore,

$$
\begin{aligned}
& \dot{V}_{k}(t)=\mathbb{E}\left[\dot{\delta}(t)^{T}\left(I_{N} \otimes P\right) \delta(t) 1_{\{\sigma(t)=k\}}+\delta(t)^{T}\right. \\
& \left.\cdot\left(I_{N} \otimes P\right) \dot{\delta}(t) 1_{\{\sigma(t)=k\}}\right]+\sum_{k=1}^{\ell} \sum_{j=1}^{\ell} q_{j k} V_{j}(t) \\
& =\mathbb{E}\left[-\mu \delta(t)^{T}\left[\left(\mathscr{M} \mathscr{L}+\mathscr{L}^{T} \mathscr{M}^{T}\right) \otimes\left(P B B^{T} P\right)\right] \delta(t)\right. \\
& \left.\cdot 1_{\{\sigma(t)=k\}}\right]+\sum_{j=1}^{\ell} q_{j k} V_{j}(t) .
\end{aligned}
$$

Accordingly,

$$
\begin{aligned}
V(t) & =\sum_{k=1}^{\ell} V_{k}(t)=\sum_{k=1}^{\ell} \mathbb{E}\left[-\mu \delta(t)^{T}\right. \\
\cdot & {\left.\left[\left(\mathscr{M} \mathscr{L}+\mathscr{L}^{T} \mathscr{M}^{T}\right) \otimes\left(P B B^{T} P\right)\right] \delta(t) 1_{\{\sigma(t)=k\}}\right] } \\
& +\sum_{k=1}^{\ell} \sum_{j=1}^{\ell} q_{j k} V_{j}(t) .
\end{aligned}
$$

Because the row sums of $\mathcal{Q}$ are zero, $\sum_{k=1}^{\ell} \sum_{j=1}^{\ell} q_{j k} V_{j}(t)=$ $\sum_{j=1}^{\ell} \sum_{k=1}^{\ell} q_{j k} V_{j}(t)=0$. This leads us to

$$
\begin{aligned}
& \dot{V}(t)=\mathbb{E}\left[-\mu \delta(t)^{T}\left(\sum_{k=1}^{\ell}\left(\mathscr{M} \mathscr{L}+\mathscr{L}^{T} \mathscr{M}^{T}\right)\right)\right. \\
& \left.\otimes\left(P B B^{T} P\right) \delta(t) 1_{\{\sigma(t)=k\}}\right] \\
& \leq-\pi^{*} \mu \mathbb{E}\left[\delta(t)^{T}\left[\left(\mathscr{L}_{\cup}+\mathscr{L}_{\cup}^{T}\right) \otimes\left(P B B^{T} P\right)\right] \delta(t)\right],
\end{aligned}
$$

because $\pi_{k} \geq \pi^{*} \forall k=1, \ldots, \ell, \sum_{k=1}^{\ell} \mathscr{L}_{k}=\mathscr{L}_{U}$ and $\mathscr{M} \mathscr{L}_{U}=$ $\mathscr{L}_{U}$. In fact, $\mathscr{L}_{U}+\mathscr{L}_{U}^{T}$ can be regarded as a Laplacian matrix of a connected undirected graph, so there exists an orthogonal matrix $U \in \mathbb{R}^{N \times N}$ such that $\mathscr{L}_{U}+\mathscr{L}_{U}^{T}=U \Lambda U^{T}$, where $\Lambda=$ $\operatorname{diag}\left\{\lambda_{i}\right\}_{i=1, \ldots, N}$ and $\lambda_{i}$ are eigenvalues of $\mathscr{L}_{U}+\mathscr{L}_{U}^{T}$, and $U=$ $\left[(1 / \sqrt{N}) \mathbf{1}_{N}, U_{2}\right]$ with $U_{2} \in \mathbb{R}^{N \times(N-1)}$. Subsequently,

$$
\begin{aligned}
\delta(t)^{T}\left[\left(\mathscr{L}_{U}+\mathscr{L}_{U}^{T}\right) \otimes\left(P B B^{T} P\right)\right] \delta(t)=\delta(t)^{T} \\
\cdot\left(U \otimes I_{n}\right)\left[\Lambda \otimes\left(P B B^{T} P\right)\right]\left(U^{T} \otimes I_{n}\right) \delta(t)=\delta(t)^{T} \\
\cdot\left(U_{2} \otimes I_{n}\right) \operatorname{diag}\left\{\lambda_{i} P B B^{T} P\right\}_{i=2, \ldots, N}\left(U_{2}^{T} \otimes I_{n}\right) \delta(t) \\
\geq \lambda_{\min }\left(\mathscr{L}_{U}+\mathscr{L}_{U}^{T}\right) \delta(t)^{T}\left(U_{2} \otimes I_{n}\right) \\
\cdot\left[I_{N-1} \otimes\left(P B B^{T} P\right)\right] \times\left(U_{2}^{T} \otimes I_{n}\right) \delta(t) \\
\geq \beta \lambda_{\min }\left(\mathscr{L}_{U}+\mathscr{L}_{U}^{T}\right) \delta(t)^{T}\left(I_{N} \otimes P\right) \delta(t) .
\end{aligned}
$$

This finally leads to

$$
\dot{V}(t) \leq-\pi^{*} \mu \beta \lambda_{\min }\left(\mathscr{L}_{U}+\mathscr{L}_{U}^{T}\right) V(t) \leq 0 .
$$

Thus, $V(t)$ converges to a stationary point at which $\dot{V}(t)=0$ due to Lasalle's invariance principle. It can be easily seen that $\dot{V}(t)=0$ if and only if $V(t)=0$. Hence, the meansquare consensus is achieved with the speed lower bounded by $\pi^{*} \mu \beta \lambda_{\min }\left(\mathscr{L}_{U}+\mathscr{L}_{U}^{T}\right)$, i.e., (7).

Theorem 4 provides a powerful consensus design for (i) the most general class of randomly switching graphs where none of them or their union is balanced; (ii) a class of heterogeneous uncertain linear MASs with state multiplicative uncertainties. These distinguish the current research from the existing literature, and hence, clearly shows its contribution.

Remark 5. The result of Theorem 4 also covers the scenario of consensus for homogeneous linear MASs under switching directed topologies where none of switched topologies nor their union is balanced and the circumstance of heterogeneous linear MASs with multiplicative uncertainties with fixed unbalanced directed topology.

3.2. Leader-Follower Consensus Design. In the leaderless consensus control, all agents converge to an unknown state due to uncertainties. This motivates the control of uncertain agents (2) to track their known nominal dynamics,

$$
\dot{x}_{0}=A x_{0} .
$$

The following assumption is utilized:

(A5): At least one follower is connected to the leader in at least one switched graph.

Then the tracking consensus design is as follows.

Theorem 6. Under assumptions (A1)-(A5), the uncertain MAS (2) converges to the leader (16) in the mean-square sense, by the distributed rank- $\alpha$ consensus controller, for any $\mu>0$, $i=1, \ldots, N$,

$$
\begin{aligned}
u_{i}(t)= & -\mu B^{T} \bigvee^{T} \widehat{P} \bigvee \sum_{j \in \mathcal{N}_{i}(t)} a_{i j}(t)\left(x_{i}(t)-x_{j}(t)\right) \\
& -a_{i 0} B^{T} \bigvee^{T} \widehat{P} \bigvee\left(x_{i}(t)-x_{0}(t)\right),
\end{aligned}
$$

where $a_{i 0}>0$ if agent $i$ is connected to the leader. Moreover, the consensus speed is lower bounded by

$$
\pi^{*} \beta \lambda_{\min }\left(\widehat{\mathscr{L}}_{\mathrm{U}}+\widehat{\mathscr{L}}_{\mathrm{U}}^{T}\right)
$$

where $\widehat{\mathscr{L}}_{U} \triangleq \mu \mathscr{L}_{U}+\operatorname{diag}\left\{a_{i 0}\right\}_{i=1, \ldots, N}$.

Proof. Denote $\xi_{i}(t) \triangleq x_{i}(t)-x_{0}(t), i=1, \ldots, N$ and $\xi(t) \triangleq$ $\left[\xi_{1}(t)^{T}, \ldots, \xi_{N}(t)^{T}\right]^{T}$. Then we have

$$
\dot{\xi}(t)=\widehat{\mathscr{A}} \xi(t)+\left(I_{N} \otimes A\right) \Delta A x(t),
$$

where $\widehat{\mathscr{A}} \triangleq I_{N} \otimes A-\widehat{\mathscr{L}} \otimes\left[B B^{T} \mathrm{~V}^{T} \widehat{P} \vee\right]$ and $\widehat{\mathscr{L}} \triangleq$ $\mu \mathscr{L}+\operatorname{diag}\left\{a_{i 0}\right\}_{i=1, \ldots, N}$. Consider the following nonnegative quadratic functions:

$$
\begin{aligned}
& V(t)=\mathbb{E}\left[\xi(t)^{T}\left(I_{N} \otimes P\right) \xi(t)\right], \\
& V_{k}(t)=\mathbb{E}\left[\xi(t)^{T}\left(I_{N} \otimes P\right) \xi(t) 1_{\{\sigma(t)=k\}}\right], \\
& k=1, \ldots, \ell .
\end{aligned}
$$


$\mathscr{G}_{1}$

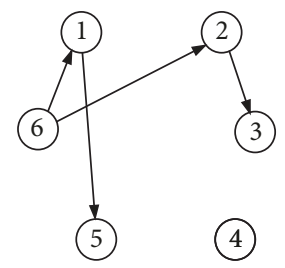

$\mathscr{G}_{2}$

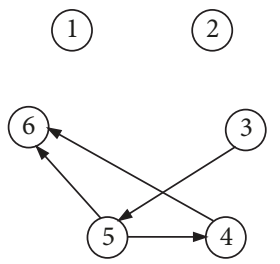

$\mathscr{G}_{\cup}$

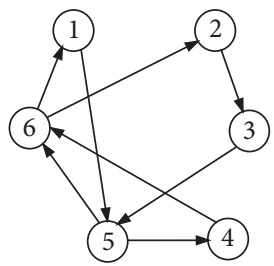

FIGURE 1: Switching topologies of leaderless MAS.
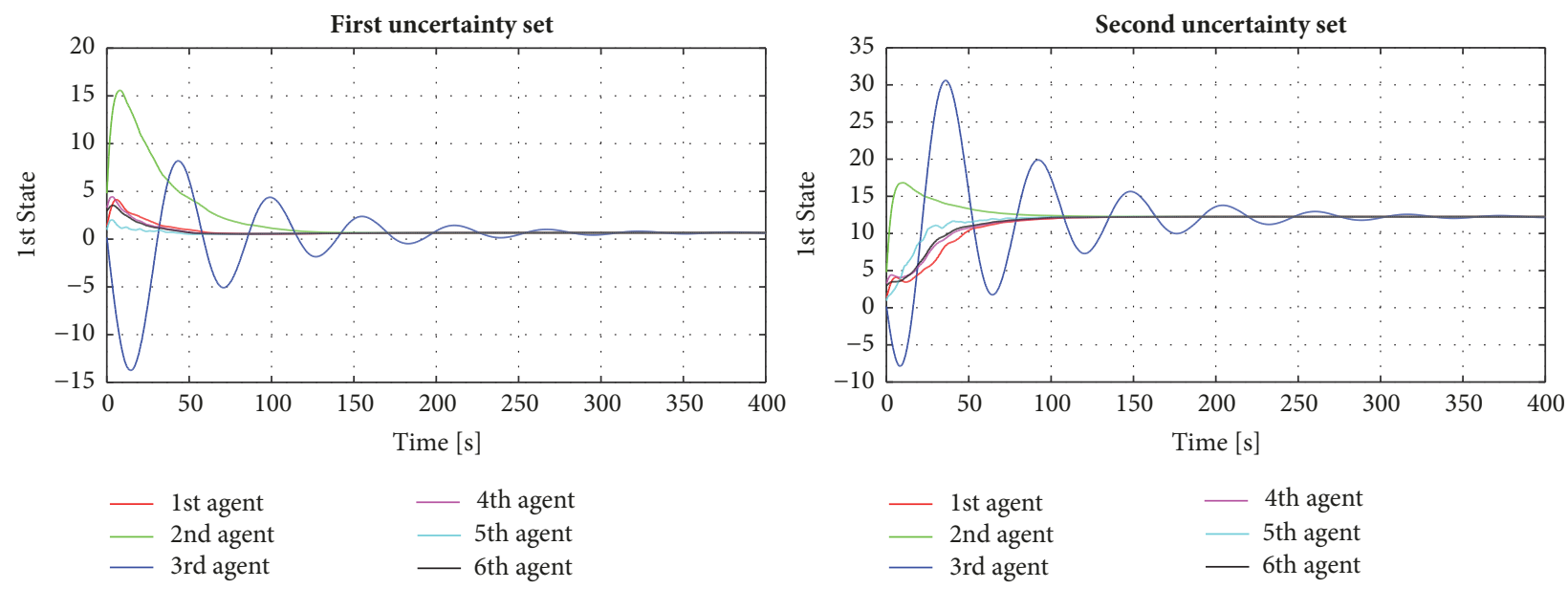

FIGURE 2: Leaderless consensus with different uncertainty sets.

Similarly to the proof of Theorem 4, we can show that

$$
\begin{aligned}
& \dot{V}_{k}(t) \\
& \leq \mathbb{E}\left[-\xi(t)^{T}\left[\left(\widehat{\mathscr{L}}+\widehat{\mathscr{L}}^{T}\right) \otimes\left(P B B^{T} P\right)\right] \xi(t) 1_{\{\sigma(t)=k\}}\right] \\
& \quad+\sum_{j=1}^{\ell} q_{j k} V_{j}(t) .
\end{aligned}
$$

Hence,

$$
\begin{aligned}
& \dot{V}(t) \\
& \quad \leq \mathbb{E}\left[-\beta \xi(t)^{T}\left(\sum_{k=1}^{\ell}\left(\widehat{\mathscr{L}}+\widehat{\mathscr{L}}^{T}\right) \otimes P\right) \xi(t) 1_{\{\sigma(t)=k\}}\right] \\
& \quad \leq-\pi^{*} \beta \mathbb{E}\left[\xi(t)^{T}\left[\left(\widehat{\mathscr{L}}_{\cup}+\widehat{\mathscr{L}}_{\cup}^{T}\right) \otimes P\right] \xi(t)\right] .
\end{aligned}
$$

Then $\widehat{\mathscr{L}}_{U}+\widehat{\mathscr{L}}_{U}^{T}=\mu\left(\mathscr{L}_{U}+\mathscr{L}_{U}^{T}\right)+\operatorname{diag}\left\{a_{i 0}\right\}_{i=1, \ldots, N}>0$ due to assumption (A5) [4], since $\mu\left(\mathscr{L}_{U}+\mathscr{L}_{U}^{T}\right)$ is the Laplacian matrix of a connected undirected graph due to assumption (A3). Accordingly, we can deduce similarly to the proof of Theorem 4 that

$$
\dot{V}(t) \leq-\pi^{*} \beta \lambda_{\min }\left(\widehat{\mathscr{L}}_{U}+\widehat{\mathscr{L}}_{\cup}^{T}\right) V(t) \leq 0
$$

Thus, according to Lasalle's invariance principle, $V(t)$ converges to a stationary point at which $\dot{V}(t)=0$ which occurs if and only if $V(t)=0$. Therefore, the mean-square consensus is achieved and the speed is lower bounded by $\pi^{*} \beta \lambda_{\min }\left(\widehat{\mathscr{L}}_{U}+\right.$ $\widehat{\mathscr{L}}_{\cup}^{T}$ ), i.e., (18).

\section{Illustrative Example}

To illustrate the proposed consensus designs, let us consider an MAS composing of 6 linear agents (2) with nominal state matrices satisfying assumptions (A1) and (A2),

$$
\begin{aligned}
& A=\left[\begin{array}{ccc}
0 & 1 & 0 \\
0 & 0 & 1 \\
0 & -2 & -3
\end{array}\right], \\
& B=\left[\begin{array}{l}
0 \\
0 \\
1
\end{array}\right] .
\end{aligned}
$$

First, the leaderless low-rank consensus design is demonstrated where the MAS topology randomly switches between two directed graphs $\mathscr{G}_{1}$ and $\mathscr{G}_{2}$ shown in Figure 1 .

Here, neither $\mathscr{G}_{1}, \mathscr{G}_{2}$, nor $\mathscr{G}_{\cup}$ is balanced and $\mathscr{G}_{\cup}$ has a spanning tree; i.e., assumption (A3) is satisfied. The random process for switched graphs is described by a continuous-time Markov chain with generator matrix $Q=\left[\begin{array}{cc}-1 & 1 \\ 1 & -1\end{array}\right]$ and the invariant distribution $\pi=[1 / 2,1 / 2]$. Model uncertainties of agents in (2) are randomly generated where $\Delta A_{i}$ are distinct diagonal random matrices such that assumption (A4) is satisfied. The design parameters are chosen as $\widehat{Q}=1, \mu=0.5$. Then the simulation results are displayed in Figure 2 with two different sets of uncertainties. Obviously, agents reach consensus but the responses of agents and the consensus state are affected by uncertainties. 

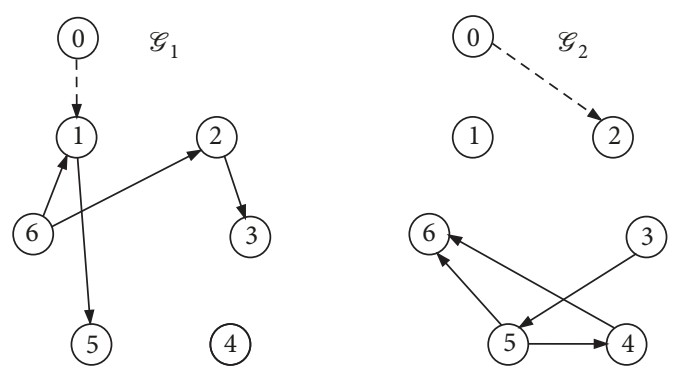

FIGURE 3: Switching topologies of leader-follower MAS.

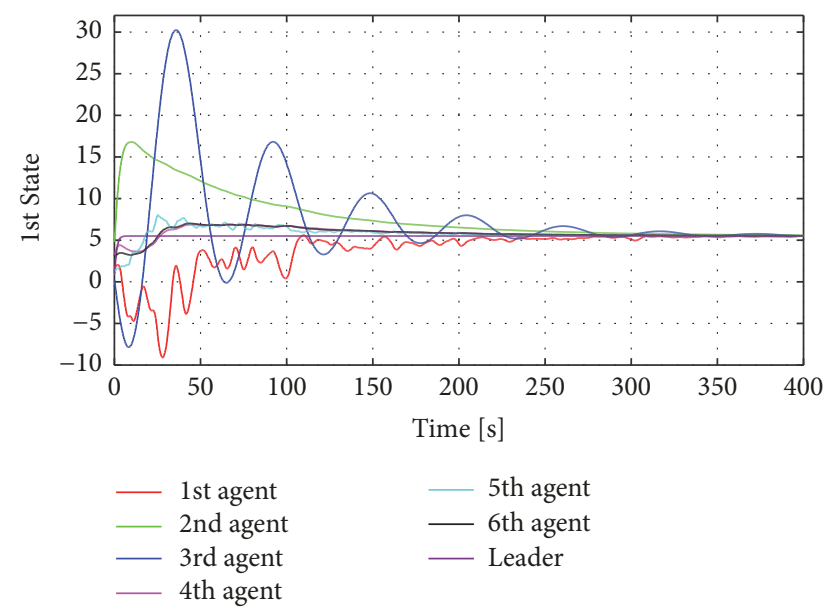

FIGURE 4: Leader-follower consensus.

Next, the proposed leader-follower low-rank consensus design is illustrated where the leader called agent 0 is connected to one follower in only one switched graph as seen in Figure 3.

Subsequently, the uncertainties are also randomly generated but design parameters are the same as above. The simulation result is then shown in Figure 4. Clearly, the consensus tracking is achieved even though the communications among them are randomly switched; none of switched graphs is connected or balanced and the union graph is unbalanced, and the leader is connected to only one follower in only one switched graph.

\section{Conclusions}

A novel approach has been proposed in this paper to design fully distributed consensus controllers for heterogeneous linear MASs with state multiplicative uncertainties and randomly switching graphs where none of them or their union is balanced. In addition, the proposed consensus controllers are low rank and have nonconservative coupling strengths. Numerical simulations were carried out to illustrate the effectiveness of the theoretical results.

The future studies should investigate more complex scenarios with more general classes of uncertainties, and when time delays, input, or state constraints exist.

\section{Data Availability}

The data used to support the findings of this study are included within the article.

\section{Conflicts of Interest}

The author declares that there are no conflicts of interest regarding the publication of this paper.

\section{References}

[1] A. Jadbabaie, J. Lin, and A. S. Morse, "Coordination of groups of mobile autonomous agents using nearest neighbor rules," IEEE Transactions on Automatic Control, vol. 48, no. 6, pp. 988-1001, 2003.

[2] R. Olfati-Saber, J. A. Fax, and R. M. Murray, "Consensus and cooperation in networked multi-agent systems," Proceedings of the IEEE, vol. 95, no. 1, pp. 215-233, 2007.

[3] F. Xiao and L. Wang, "Consensus problems for high-dimensional multi-agent systems," IET Control Theory and Applications, vol. 1, no. 3, pp. 830-837, 2007.

[4] Z. Li, Z. Duan, G. Chen, and L. Huang, "Consensus of multiagent systems and synchronization of complex networks: a unified viewpoint," IEEE Transactions on Circuits and Systems I: Regular Papers, vol. 57, no. 1, pp. 213-224, 2010.

[5] C.-Q. Ma and J.-F. Zhang, "Necessary and sufficient conditions for consensusability of linear multi-agent systems," IEEE Transactions on Automatic Control, vol. 55, no. 5, pp. 1263-1268, 2010.

[6] Z. Li, G. Wen, Z. Duan, and W. Ren, "Designing fully distributed consensus protocols for linear multi-agent systems with directed graphs," IEEE Transactions on Automatic Control, vol. 60, no. 4, pp. 1152-1157, 2015.

[7] M. Huang, S. Dey, G. N. Nair, and J. H. Manton, "Stochastic consensus over noisy networks with Markovian and arbitrary switches," Automatica, vol. 46, no. 10, pp. 1571-1583, 2010.

[8] K. You, Z. Li, and L. Xie, "Consensus condition for linear multiagent systems over randomly switching topologies," Automatica, vol. 49, no. 10, pp. 3125-3132, 2013.

[9] N. Chopra and M. W. Spong, "Passivity-based control of multiagent systems," in Advances in Robot Control: From Everyday Physics to Human-Like Movements, S. Kawamura and M. Svinin, Eds., pp. 107-134, Springer-Verlag, New York, NY, USA, 2006.

[10] S. Su and Z. Lin, "Distributed consensus control of multi-agent systems with higher order agent dynamics and dynamically changing directed interaction topologies," IEEE Transactions on Automatic Control, vol. 61, no. 2, pp. 515-519, 2016.

[11] Y. Wang, L. Cheng, W. Ren, Z.-G. Hou, and M. Tan, "Seeking consensus in networks of linear agents: communication noises and Markovian switching topologies," IEEE Transactions on Automatic Control, vol. 60, no. 5, pp. 1374-1379, 2015.

[12] L. Moreau, "Stability of multiagent systems with time-dependent communication links," IEEE Transactions on Automatic Control, vol. 50, no. 2, pp. 169-182, 2005.

[13] W. Ren and R. W. Beard, "Consensus seeking in multiagent systems under dynamically changing interaction topologies," IEEE Transactions on Automatic Control, vol. 50, no. 5, pp. 655661, 2005.

[14] D. H. Nguyen, T. Narikiyo, and M. Kawanishi, "Low-rank distributed consensus controller design for linear multi-agent systems under randomly switching directed topologies and 
model uncertainties," in Proceedings of the 2017 IFAC World Congress, 2017.

[15] Y. Cao and W. Ren, "Optimal linear-consensus algorithms: An lqr perspective," IEEE Transactions on Systems, Man, and Cybernetics, Part B: Cybernetics, vol. 40, no. 3, pp. 819-830, 2010.

[16] H. Zhang, F. L. Lewis, and A. Das, "Optimal design for synchronization of cooperative systems: state feedback, observer and output feedback," IEEE Transactions on Automatic Control, vol. 56, no. 8, pp. 1948-1952, 2011.

[17] D. H. Nguyen and S. Hara, "Hierarchical decentralized stabilization for networked dynamical systems by LQR selective pole shift," in Proceedings of the 19th IFAC World Congress on International Federation of Automatic Control, IFAC 2014, pp. 5778-5783, August 2014.

[18] K. H. Movric and F. L. Lewis, "Cooperative optimal control for multi-agent systems on directed graph topologies," IEEE Transactions on Automatic Control, vol. 59, no. 3, pp. 769-774, 2014.

[19] D. Madjidian and L. Mirkin, "Distributed control with lowrank coordination," IEEE Transactions on Control of Network Systems, vol. 1, no. 1, pp. 53-63, 2014.

[20] D. H. Nguyen, "A sub-optimal consensus design for multi-agent systems based on hierarchical LQR," Automatica, vol. 55, pp. 8894, 2015.

[21] D. H. Nguyen, "Reduced-order distributed consensus controller design via edge dynamics," Institute of Electrical and Electronics Engineers Transactions on Automatic Control, vol. 62, no. 1, pp. 475-480, 2017.

[22] M. D. Fragoso and O. L. Costa, "A unified approach for stochastic and mean square stability of continuous-time linear systems with Markovian jumping parameters and additive disturbances," SIAM Journal on Control and Optimization, vol. 44, no. 4, pp. 1165-1191, 2005.

[23] L. Cheng, Z.-G. Hou, Y. Lin, M. Tan, and W. J. Zhang, "Solving a modified consensus problem of linear multi-agent systems," Automatica, vol. 47, no. 10, pp. 2218-2223, 2011.

[24] Y. Shang, "Couple-group consensus of continuous-time multiagent systems under Markovian switching topologies," Journal of The Franklin Institute, vol. 352, no. 11, pp. 4826-4844, 2015. 


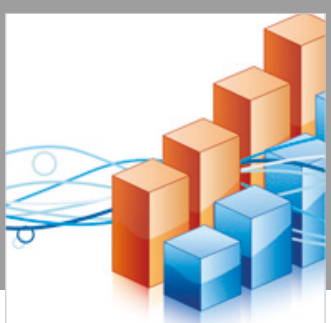

Advances in

Operations Research

\section{-n-m}
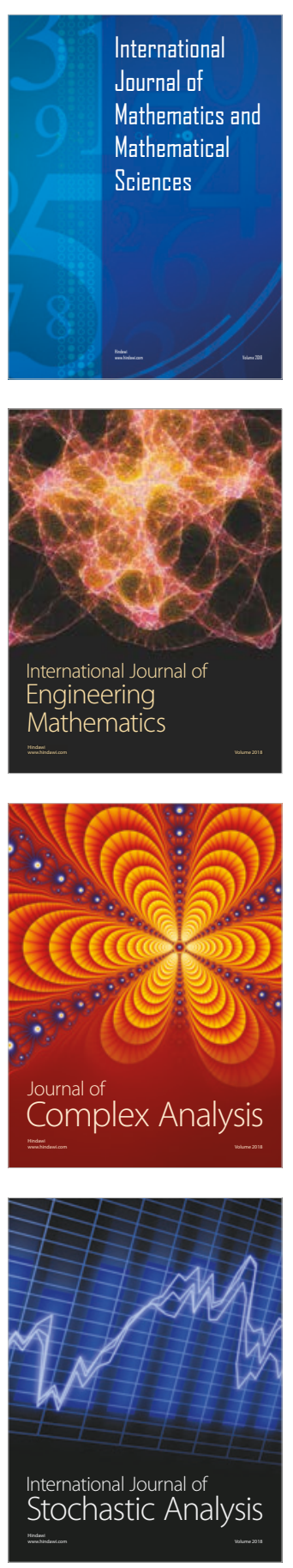
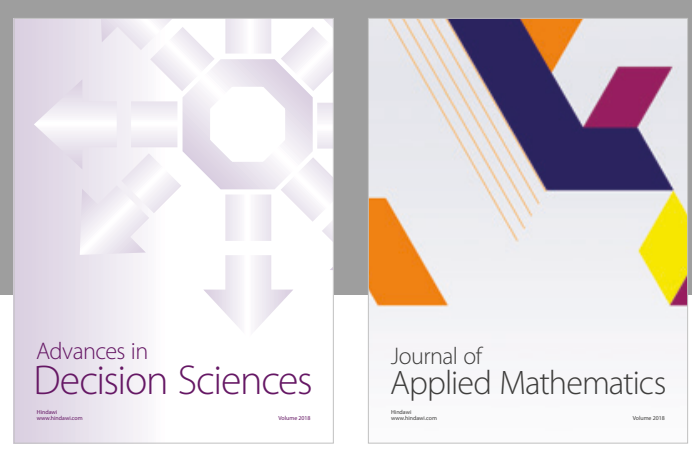

Journal of

Applied Mathematics
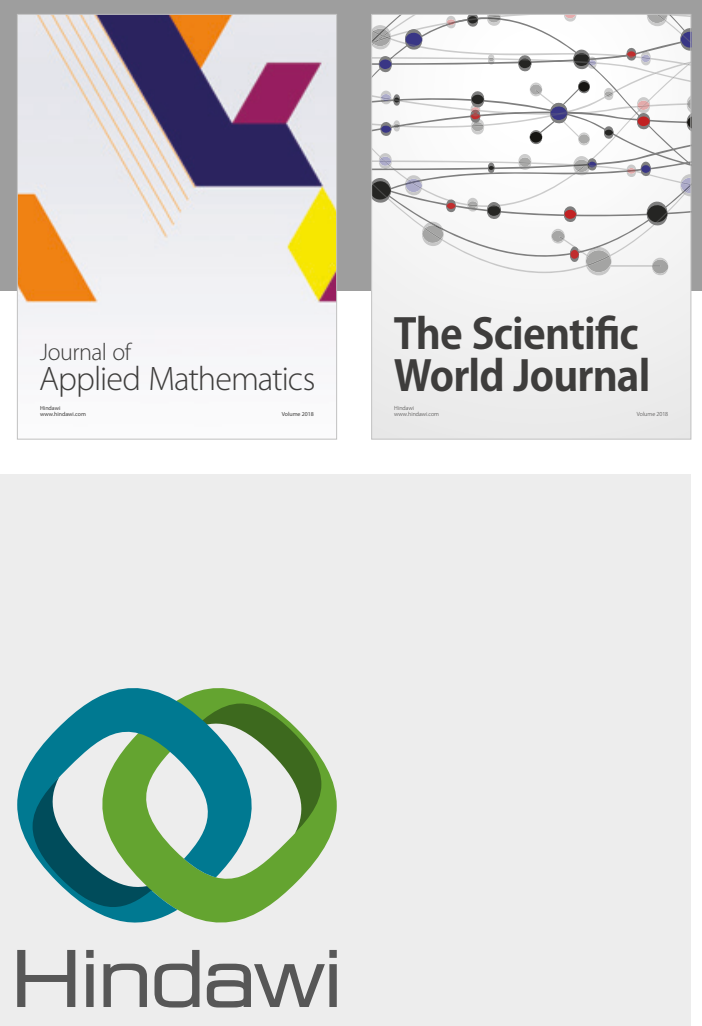

Submit your manuscripts at

www.hindawi.com

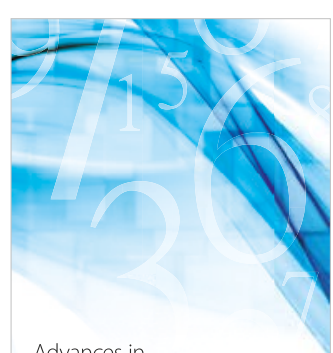

Advances in
Numerical Analysis
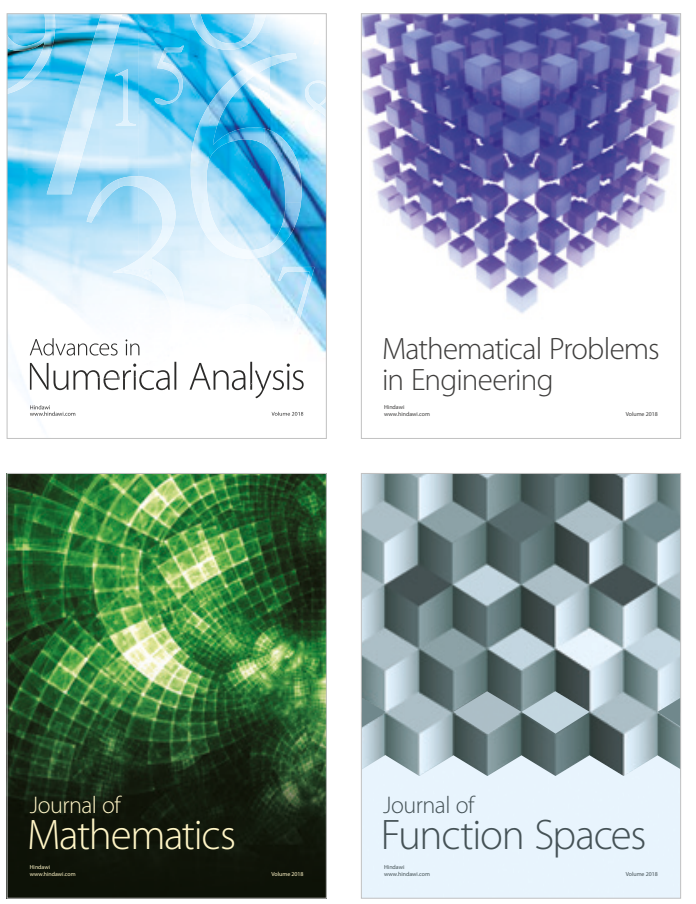

Mathematical Problems in Engineering

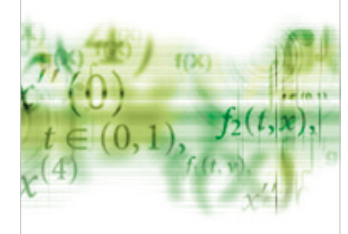

International Journal of

Differential Equations

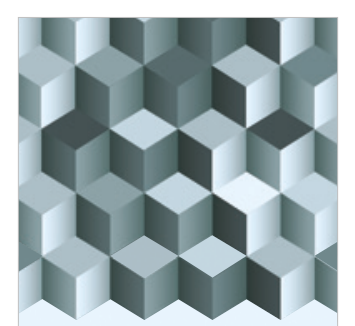

Journal of

Function Spaces

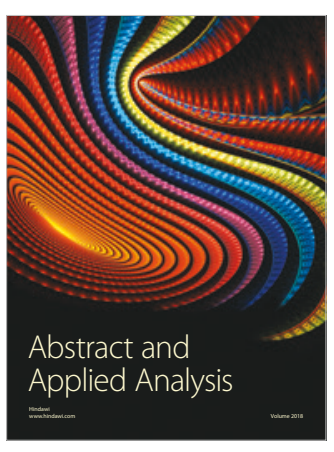

The Scientific

World Journal

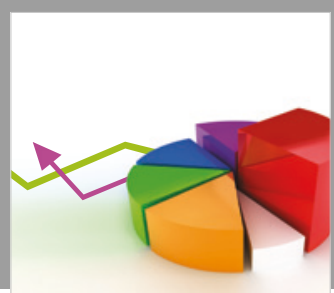

Journal of

Probability and Statistics
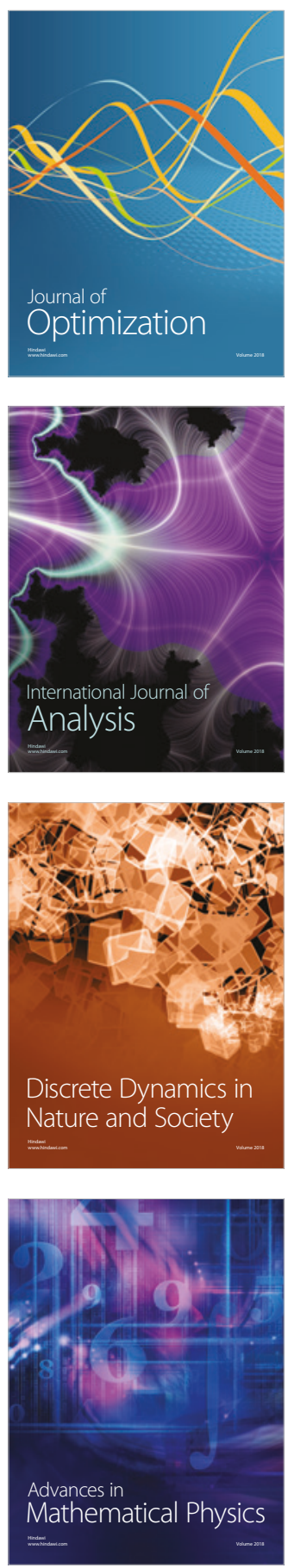